\title{
Does macular laser alter the refraction in a diabetic with maculopathy? A pilot study
}

\begin{abstract}
Purpose To determine whether macular photocoagulation has any effect on refraction in diabetics.

Methods This was a prospective study of 12 eyes of 12 diabetics with refraction at 2 weeks before and at 4 weeks and 4 months after argon laser. This was combined with a questionnaire survey of attitude among ophthalmologists on prescribing spectacles for diabetics awaiting macular laser. The main outcome measure was the spherical equivalents (SEQ) before and after treatment. Results Only $36 \%$ of ophthalmologists surveyed would prescribe corrective lenses in patients waiting for macular photocoagulation. The median change in magnitude of SEQ at 4-6 weeks post-laser compared to pre-laser was $0.36 \mathrm{D}$ (interquartile range 0.18-0.50 D). The median change in SEQ at 4 months was $0.25 \mathrm{D}$ (interquartile range $0.12-0.56 \mathrm{D})$. These changes in SEQ from pretreatment values were not statistically significant on Wilcoxon signed-rank test ( $p=0.17$ and 0.10 , respectively). The

Many of these patients have had difficulty reading or have experienced a change in their refraction since their last optometric/ ophthalmic assessment. However, it is our experience that a common practice among health care providers in the UK is to delay prescription of new lenses until maculopathy has been 'treated'. To test this suspicion, we performed a questionnaire survey of consultant ophthalmologists in our Region (Trent).

As far as we are aware, there has been no study documenting a significant change of refraction as a result of macular photocoagulation alone. If such treatment does not alter refractive status to any practical extent, spectacles can be prescribed without delay whenever required, even on detection of diabetic maculopathy.

In order to address the possible change in refraction from macular photocoagulation, we conducted a prospective controlled study on a group of diabetic patients who were scheduled for photocoagulation in order to treat diabetic maculopathy.
\end{abstract} correlation coefficient between difference and average SEQ was $0.02(95 \% \mathrm{CI}-0.14$ to 0.19 , $p=0.75)$ at 4 weeks and $0.11(95 \%$ CI -0.06 to $0.275, p=0.17$ ) at 4 months, indicating no tendency for change in refraction with increasing ametropia.

Conclusion This pilot study implies that argon laser photocoagulation in diabetic maculopathy does not alter refraction. Prescription of corrective lenses can be performed prior to laser therapy, if required.

Key words Diabetic maculopathy, Photocoagulation, Refraction

Diabetic maculopathy, a common condition affecting up to a quarter of the diabetic population, ${ }^{1}$ is often diagnosed by optometrists ${ }^{2}$ and referred to hospital ophthalmic departments where appropriate photocoagulation treatment ${ }^{3}$ can be performed.

\section{Methods}

The qeustionnaire survey was carried out by a postal method. All 59 consultant

ophthalmologists from Trent Region were sent a questionnaire (see Appendix).

Twelve consecutive subjects for the refraction study were recruited from the diabetic eye clinic at Queen's Medical Centre in Nottingham, UK between August 1999 and October 1999. Informed consent was obtained from the patients who satisfied all the following entry criteria:

1. Untreated clinically significant maculopathy in one or both eyes. ${ }^{3}$

2. No other macular disease.

3. No patient factors (language, mental difficulties) which might affect subjective refraction.
K.T. Sim

L. Tong

S.A. Vernon

Department of

Ophthalmology

University Hospital

Nottingham NG7 2UH, UK

S.A. Vernon, DM, FRCS, FRCOphth

The Eye and ENT Centre Queens Medical Centre Nottingham NG7 2UH, UK

Tel: +44 (0)1159249924,

ext 43200

Fax: +44 (0)1159709749

e-mail:

Stephen.vernon@mail.

qmcuh-trent.nhs.uk

Received: 14 November 2000 Accepted in revised form: 30 April 2001 
We excluded patients with proliferative diabetic retinopathy or severe non-proliferative retinopathy who might require panretinal photocoagulation (PRP) in the near future.

All subjects had undergone a medical and ophthalmic evaluation, including slit-lamp biomicroscopy and contact lens examination where necessary, to document the extent of the macular oedema. In our photocoagulation protocol, areas of retinal thickening (including circinate exudates) were treated with 50-100 $\mu \mathrm{m}$ argon laser burns of $0.1 \mathrm{~s}$ duration at least one spot size apart. Laser power was increased until a visible blanching was observed on the retina. The indication for treatment in our unit is the presence of clinically significant macular oedema (CSMO) as defined in ETDRS $^{3}$ and does not include a reduction in acuity.

Baseline non-cycloplegic refractions were performed at 2 weeks before laser. Subsequent refractions were performed at 4-6 weeks after laser and again at 4 months after laser. Standard refraction steps ${ }^{4}$ were adhered to in all cases and carried out in the same room under standard lighting conditions. Briefly, the subject's unaided and pinhole visual acuities on a standard Snellen chart were first determined. This was followed by retinoscopy under dimmed light at the adopted working distance of the refractionist of $0.5 \mathrm{~m}$. Subjective verification was performed by first refining the spherical correction to the last line seen clearly. Cylinder axis and power were checked using a crossed-cylinder technique by directing the subjects to look at a circular letter near their acuity limit. The duochrome test was used for binocular balancing and the same illuminated Snellen chart, pinhole/occluder, trial-frame, trial lens set, retinoscope and crossed-cylinder were employed in all refractions. Interobserver difference was eliminated by employing the same refractionist throughout the study with results of baseline refractions being masked at follow-up visits in an attempt to minimise bias. Nonphotocoagulated fellow eyes of patients with unilateral CSMO were used as controls. Using slit-lamp biomicroscopy, we assessed each patient's fundus for resolution of oedema at 3 months after photocoagulation. The patient's initial refractive error was classified as in the Beaver Dam Eye Study, ${ }^{5}$ where myopia was defined as refractive error less than $-0.50 \mathrm{D}$ and hyperopia, more than $+0.50 \mathrm{D}$.

The main outcome measure was the change in spherical equivalent (SEQ) in dioptres (after treatment minus before treatment). The change in SEQ for subjects was analysed using the non-parametric Wilcoxon signedrank test in the SPSS (version 9.0) statistical software package. A two tailed $p$ value of $<0.05$ was considered as statistically significant. Bland-Altman analyses ${ }^{6}$ were also performed by plotting the difference in SEQ (prelaser minus post-laser values) against the mean value for each eye.
Table 1. Spherical equivalents (SEQ) of 12 subjects at 2 weeks prelaser, 4-6 weeks post-laser and 4 months post-laser

\begin{tabular}{cccc}
\hline Subject no. & Pre-laser & 1st post-laser & 2nd post-laser \\
\hline 1 & +0.375 & +0.125 & +0.25 \\
2 & -0.625 & -0.125 & -0.375 \\
3 & 0 & -0.625 & -0.125 \\
4 & +0.25 & +0.375 & +0.25 \\
5 & 0 & -0.5 & -0.5 \\
6 & +2.5 & +2 & +2.5 \\
7 & +0.875 & +1 & +0.625 \\
8 & -1.5 & -2.125 & -1.75 \\
9 & -4 & -4.25 & -5 \\
10 & +2.125 & +1.75 & +1.5 \\
11 & -0.375 & 0 & 0 \\
12 & +0.875 & +1 & +1 \\
\hline
\end{tabular}

\section{Results}

Thirty-nine of the 59 consultants of Trent Region returned the postal questionnaire (69\% response rate). Thirty-six per cent would prescribe whilst a patient was waiting for macular laser, $23 \%$ would wait until 2 weeks after macular laser while $41 \%$ would delay prescription for 3 months after macular photocoagulation.

Twelve eyes of 12 diabetics (6 non-insulin-dependent and 6 insulin-dependent) were included in the refraction study. There were 8 female and 4 male subjects. The median age of the subjects was 59.5 years (interquartile range 46-68.5) with a median duration of diabetes of 10.5 years (interquartile range 2.5-16.5). Five subjects were emmetropic, 3 were myopic and 4 hyperopic at baseline pre-laser refraction. The visual acuity for subjective refraction throughout the study recorded a mean acuity of $6 / 9$ (range $6 / 4$ to $6 / 18$ ). Each eye received an average of 92 (SD 105) burns (either focal or grid). There were 8 patients with unilateral CSMO and their fellow eyes were used as controls. At 3 months after photocoagulation, slit-lamp biomicroscopy revealed all but 1 patient to have a dry macula. The patient who had suspected residual oedema (subject 8 in Table 1) responded to further macular photocoagulation at 4 months follow-up.

Table 1 shows the SEQ of the refraction at various times during the study for the photocoagulated eyes. The median change in absolute magnitude of SEQ was $0.36 \mathrm{D}$ at 4-6 weeks post-laser (interquartile range 0.18-0.50 D). With the Wilcoxon signed-rank test, there was no statistically significant change in refraction at 4-6 weeks following laser photocoagulation ( $p=0.17)$. At 4 months post-laser, the median SEQ absolute magnitude change

Table 2. Spherical equivalents (SEQ) of control eyes at 2 weeks prelaser, at 4 weeks post-laser and 4 months post-laser

\begin{tabular}{clcc}
\hline Subject & Pre-laser & 1st post-laser & 2nd post-laser \\
\hline 1 & +0.25 & +0.125 & +0.375 \\
2 & -0.625 & -0.25 & -0.375 \\
3 & -0.375 & -1.125 & -0.375 \\
6 & $+2: 25$ & +1.75 & +2.375 \\
7 & -0.25 & +1.125 & +0.625 \\
9 & -5.5 & -5.5 & -6 \\
10 & +1.75 & +1.25 & +1.5 \\
12 & +0.875 & +1.375 & +0.75 \\
\hline
\end{tabular}




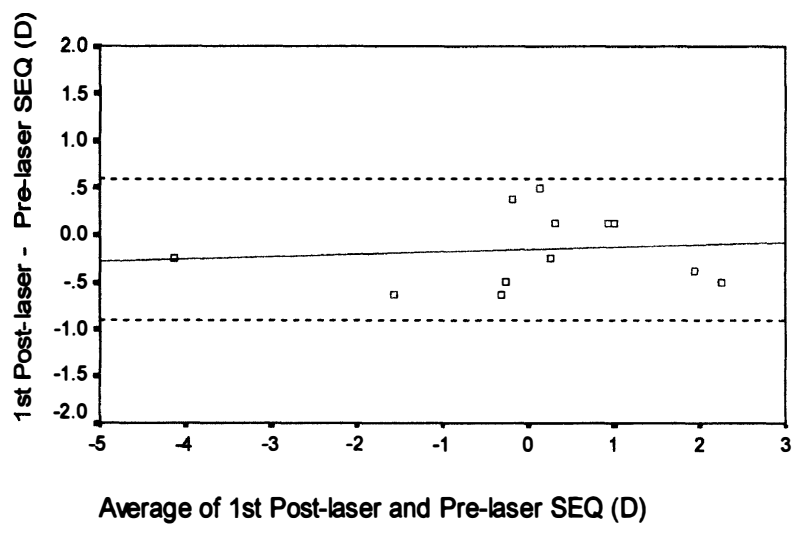

Fig. 1. Scatter-plot of the difference between the 1st post-laser SEQ and baseline SEQ against their average with $95 \%$ limits and regression line

was $0.25 \mathrm{D}$ (interquartile range of $0.12-0.56$ ). There was also no statistically significant change in SEQ between pre-laser and second post-laser refraction $(p=0.10)$. In the 8 control eyes (Table 2 ), the refraction did not change significantly during the follow-up period on Wilcoxon signed rank test $(p=0.67)$. Bland-Altman plots of SEQ difference (post-laser minus pre-laser SEQ) against average of follow-up and baseline SEQ are shown in Figs. 1 and 2 . The correlation coefficient for first follow-up data was $0.02(95 \% \mathrm{CI}-0.14$ to $0.19, p=0.75)$ and that of the second follow-up was $0.11(95 \%$ CI -0.06 to 0.28 , $p=0.17)$. Both correlation coefficients are very close to zero, confirming that there was no tendency for alteration in refraction with initial ametropia.

\section{Discussion}

Our questionnaire survey confirmed that the majority of consultant ophthalmologists would defer prescription of spectacles until after diabetic patients have received argon laser treatment for diabetic maculopathy. One can imagine that, in some cases, the management of diabetic maculopathy can result in delays for optimal refractive correction of over a year following initial diagnosis. This

\section{อ}

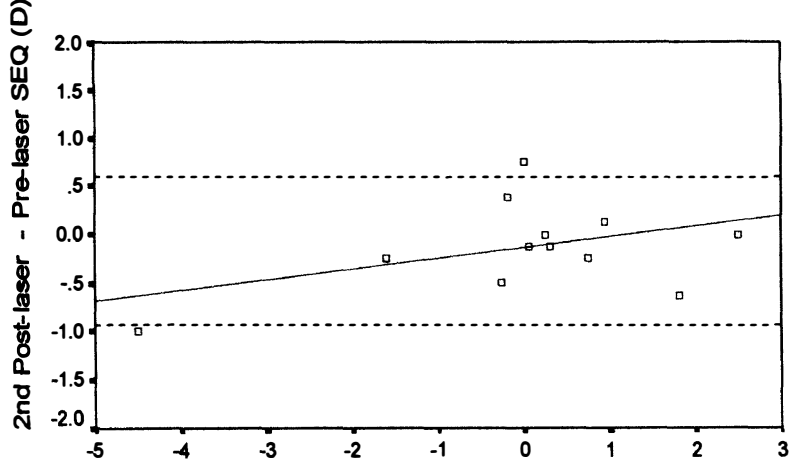

Average of 2nd Post-laser and Pre-laser SEQ (D)

Fig 2. Scatter-plot of the difference between the 2nd post-laser SEQ and pre-laser SEQ against their average with $95 \%$ limits and regression line. has important implications for patients whose newly corrected distance visual acuity would have allowed them to drive, as field loss from bilateral macular laser is almost never significant enough to result in the patient failing the DVLA field regulation. ${ }^{7}$

The refraction study was of prospective design with uniform test conditions and treatment. There are some limitations, however, including the small subject numbers and a relatively short follow-up period. Although our study was only a pilot, we nevertheless found no significant change in refractive error following laser treatment in diabetic maculopathy. We excluded patients who had pan-retinal photocoagulation as this can potentially cause temporary cycloplegia ${ }^{8}$ which might influence the refraction. In addition, our study only includes type 2 diabetics, but it is this subgroup who constitute the majority of patients requiring macular treatment alone.

Our results are consistent with the scarce literature that is partially relevant to the context of the hypothesis in our study. Reversal of retinal thickening in diabetic macular oedema 4 months after laser photocoagulation has been documented quantitatively. ${ }^{9}$ It could be argued that there might be a theoretical shift towards myopia as retinal thickness is reduced after macular argon photocoagulation. It has been shown that a decrease in retinal thickness at the posterior pole is correlated with increasing axial myopia. ${ }^{10}$ In central serous retinopathy where there is an accumulation of fluid in the macular subretinal space leading to sensory retinal detachment, refractive error can increase by up to $1 \mathrm{D} .{ }^{11}$ Such evidence may suggest a possible relationship between refractive status and changes in retinal thickness induced by pathology. On the other hand, one study showed no correlation between refraction and duration of type 1 diabetes. ${ }^{12}$ However, the authors of this study had not analysed the correlation between extent of oedema and refraction.

The spherical equivalent (SEQ), as used in our study, is a measure of the position of the focal point of the light rays on the retina. In an optical system with an astigmatic property, this would indicate the position of the circle of least confusion on the conoid of Sturm. ${ }^{4}$ Assuming that the light rays are focused on the photoreceptors, the SEQ (and the location of this optical point) can be used to detect a change in the position of the foveal photoreceptor layer rather than the thickness of the entire neural retina. Diabetic maculopathy is characterised by the accumulation of extracellular fluid in Henle's layer (outer plexiform layer) of the retina. ${ }^{13}$ The axial position of the photoreceptors should not be altered in diabetic macular oedema and therefore there should not be any change in SEQ. This concept is in keeping with our results and with our rejection of the common belief that retinal oedema alters refractive error.

Although the sample was small, our pilot study does cover a wide age range and includes both insulin and non-insulin-dependent diabetics. Our cohort of patients appears to be representative of patients undergoing macular laser treatment. This pilot study could be 
extended to a future definitive larger study, including patients who have both macular oedema as well as proliferative diabetic retinopathy. If a larger study were to be conducted, the chances of obtaining a statistically significant SEQ change, albeit small, would be higher. However, any SEQ change of $0.5 \mathrm{D}$ or less would probably be clinically insignificant, and would be unlikely to merit a change of spectacle prescription.

This pilot study suggests that there is no significant change in refractive error from macular laser photocoagulation. In the light of our findings, we suggest that it is acceptable practice to prescribe for a distance refractive error as and when required irrespective of macular laser treatment

\section{Appendix}

Suppose you have a diabetic patient seeing 6/12 with his current glasses of two years old. After new refraction, he can now see $6 / 6$. The patient is driving and is waiting for a macular photocoagulation treatment in 2 weeks time. Would you prescribe glasses: (please tick in one box)

1. Immediately?

2. Two weeks after macular laser?

3. Three months after macular laser?

\section{References}

1. Klein R, Klein BE, Moss SE. The long-term incidence of macular edema. The Wisconsin Epidemiologic Study of Diabetic Retinopathy XV. Opthalmology 1995;102:7-16.
2. Hammond CJ, Shackleton J, Flanagan DW. Comparison between an ophthalmic optician and an ophthalmologist in screening for diabetic retinopathy. Eye 1996;10:107-12.

3. Early Treatment of Diabetic Retinopathy Study Research Group. Photocoagulation of diabetic macular edema. Arch Ophthalmol 1985;103:1796-806.

4. D Abrams. Duke-Elder's practice of refraction. 10th ed. London: Churchill-Livingstone, 1993.

5. Wang Q, Klein BE, Moss SE. Refractive status in the Beaver Dam Eye Study. Study. Invest Ophthalmol Vis Sci 1994;35:4344-7.

6. Bland JM, Altman DG. Statistical methods for assessing agreement between two methods of clinical measurement. Lancet 1986;I:307-10.

7. Tong L, Vernon SA. Passing the DLVA field regulations following bilateral macular photocoagulation in diabetics. Eye 2000;14:35-8.

8. Braun CI, Benson WE, Remaley NA, Chew EY, Ferris FL III Early Treatment of Diabetic Retinopathy Study Research Group. Accommodative amplitudes in the Early Treatment Diabetic Retinopathy Study. ETDRS report 21. Retina 1995;15:275-81.

9. Shahidi M, Ogura Y, Blair NP, Zeimer R. Retinal thickness change after focal laser treatment of diabetic macular oedema. Br J Ophthalmol 1994;78:827-30.

10. Kremser B, et al. Retinal thickness analysis in subjects with different refractive conditions. Ophthalmologica 1999;213:376-9.

11. Keller JT, Polse KA. Central serous retinopathy with transitory monocular hypermetropia: a case report. Am J Optom Arch Am Acad Optom 1972;49:793-6.

12. Sjolie AK, Mortensen KK. Visual acuity and refraction in type 1 diabetic patients aged 25-34 years. Acta Ophthalmol (Copenh) 1991;69:552-4.

13. Tso MOM. Pathological study of cystoid macular oedema. Trans Ophthalmol Soc UK 1980;100:408-13. 